\title{
„Theuerste Freunde“ \\ Dresdner Intelligenz in der DDR auf der Suche nach dem bildungsbürgerlichen Erbe
}

\author{
von \\ MICHAEL KUNZ
}

Wie andernorts gestand die offizielle Kulturpolitik auch in Dresden der sogenannten Intelligenz ${ }^{1}$ Freiräume in Form von Klubs und ähnlich organisierten Einrichtungen zu. Regulär waren diese beim „Deutschen Kulturbund“ (später „Kulturbund der DDR") angesiedelt. Nun fanden sich in Dresden gleich zwei außergewöhnlich zu nennende Institutionen, deren Besonderheit wohl darin besteht, dass sie singuläre Erscheinung waren, nicht zuletzt im Hinblick auf die Intentionen, die hinter ihrer Gründung standen. Zum einen ist dies der von 1956 bis 1972 existierende „Dresdner Klub“, zum anderen der in den 1980er-Jahren gegründete „Carus-Club“ 2 , der sich nahezu ausschließlich an eine für das System höchst sensible Berufsgruppe wandte: die Ärzteschaft. Gemeinsam ist beiden zeitlich versetzt existierenden Einrichtungen trotz erheblicher Unterschiede - so viel sei vorweggenommen - ein Bekenntnis zur bildungsbürgerlichen Kultur und Tradition.

Zuvorderst wird die Untersuchung in historischer Absicht als Beitrag zur sächsischen Regionalgeschichte geführt; es sollen vornehmlich Eckpunkte der Entwicklung beider Institutionen - eine detaillierte Bearbeitung steht noch aus aufgezeigt werden. Eine ergänzende Perspektive verspricht zudem ein soziologischer Befund, der etwa der Frage nachgeht, ob und in welchem Grade von Autonomie und Selbstbestimmung der Institutionen ausgegangen werden kann oder welche Aussagen sich durch eine sozialstatistische Analyse der Mitgliederstruktur treffen lassen. Der Aufsatz versteht sich ebenfalls als Beitrag zur Diskussion um die derzeit vielbemühte ,bürgerliche Kultur' und berührt damit, so hofft der Ver-

${ }^{1}$ Eine verbindliche Definition von ,Intelligenz' lässt sich nicht geben. Hauptkriterium für die amtliche Berufs-Zuordnung war ein akademischer Abschluss. Die Kategorie Intelligenz in sich war nochmals unterteilt in Unterkategorien, so etwa die ,technische', die ,medizinische“ Intelligenz etc., wobei die Zuordnung einzelner Berufe in dieser Hinsicht großzügig gehandhabt wurde. Vgl. dazu: Alfred LeUTweIN, Die „Technische Intelligenz“ in der Sowjetischen Besatzungszone (Bonner Berichte aus Mittel- und Ostdeutschland), Bonn 1953; MANFRED LÖTSCH, Zur Entwicklung der Intelligenz in der Deutschen Demokratischen Republik, in: Ders. u. a., Die Intelligenz in der sozialistischen Gesellschaft (Schriftenreihe Soziologie), Berlin (Ost) 1980, S. 89-118.

2 Die Schreibweise ,Klub` oder ,Club` richtet sich nach der jeweils offiziellen Verwendung. 
fasser, das Interesse der beiden angeführten wissenschaftlichen Disziplinen gleichermaßen.

Thesenhaft sei dem Beitrag folgende Vermutung vorangestellt: Es kann von einem Überdauern bildungsbürgerlicher Kultur und Tradition auch in der DDR ausgegangen werden, vor allem in refugienähnlichen Sonderräumen verschiedener Institutionen oder Professionen (etwa der Ärzteschaft) ${ }^{3}$ - abgelöst von der einst konstituierenden sozialen Trägerschicht. Besonders sei dies in Dresden zu beobachten, wie unter anderem der Soziologe Karl-Siegbert Rehberg postuliert. ${ }^{4}$

Aus historischer Sicht ist das Thema „Klubs der Intelligenz in der DDR“ kaum erforscht, ${ }^{5}$ ebenso wie die weit verzweigte Institution „Kulturbund“. ${ }^{6}$ Dies verwundert umso mehr, als der ehemalige Kulturbund der DDR mit republikweit einigen hunderttausend Mitgliedern keineswegs ein Nischenphänomen darstellte. Zunächst gilt es, in gebotener Kürze, eine notwendige Begriffsklärung sowie einen sozial-historischen Überblick zu geben.

\section{Exkurs, bürgerliche Kultur' und die, Intelligenz' im Staatssozialismus}

\section{Bürgerliche Kultur}

Die vor allem in Deutschland unter Bildungsbürgertum erfassten Berufsgruppen der höheren Beamten, Ärzte, protestantischen Pfarrer, Rechtsanwälte und Richter, Gymnasiallehrer und Professoren, Schriftsteller, Verleger, Künstler und Kunstkritiker, aber auch der Naturwissenschaftler, Diplom-Ingenieure und qualifizierten Experten in großen Unternehmen etc. - Personen also mit tendenziell akademischer Bildung - bilden neben der Bourgeoisie einen weiteren Kern des Bürgertums. ${ }^{7}$ Das grundlegende Merkmal, der qualitative Fluchtpunkt des (sich

3 Exemplarisch: GÜNTER WirTh, Zu Potsdam und anderswo. Kontinuitäten des Bildungsbürgertums in der DDR, in: Manfred Hettling/Bernd Ulrich (Hg.), Bürgertum nach 1945, Hamburg 2005, S. 85-110; PAUl KAISER, Die „Erfurter Ateliergemeinschaft“ - ein bürgerlicher Kunstverein im DDR-Staatssozialismus?, in: Karl-Siegbert Rehberg/Paul Kaiser (Hg.), Abstraktion im Staatssozialismus. Feindsetzungen und Freiräume im Kunstsystem der DDR, Weimar 2003, S. 149-175; DERS., Bürgerlichkeit ohne Bürgertum?, in: Aus Politik und Zeitgeschichte. Beilage zur Wochenzeitung Das Parlament 2008, Nr. 9-10, S. 26-32.

${ }^{4} \mathrm{Vgl}$. Karl-Siegbert Rehberg, Metamorphosen des Bürgertums. Reflexionen angesichts der Dresdner Entwicklung vom Residenzbürgertum zum Refugiumsbürgertum, in: Dresdner Hefte 93 (2008), S. 90-97.

5 Vgl. unter anderem ANDREAs Zimmer, Der Leipziger Klub der Intelligenz „Gottfried Wilhelm Leibnitz“ im Kulturbund der DDR. Funktion und Arbeitsweise in den 80er Jahren (Hefte zur DDR-Geschichte 92), Berlin 2005.

6 Überblickhaft vgl. Helmut MEIER, Der Kulturbund im politischen System der DDR in den siebziger Jahren (Hefte zur DDR-Geschichte 62), Berlin 2000.

7 Vgl. JürgEn KocKA, Das europäische Muster und der deutsche Fall, in: Ders. (Hg.), Bürgertum im 19. Jahrhundert - Deutschland im europäischen Vergleich, Bd. 1: Einheit und 
im 18. und 19. Jahrhundert noch ständisch vergesellschafteten) Bildungsbürgertums war Bildung. Sie begründet auch dessen relative Rekrutierungsoffenheit. Vor allem der Besitz von Bildungswissen, sicherlich in Verbindung mit entsprechenden Fähigkeiten der Anwendung, sowie das persönliche Streben entschieden maßgeblich über Partizipationschancen. Nun war die bürgerliche Vergesellschaftung nicht zu einem geringen Teil erst aufgrund eines gemeinsam geteilten kulturellen Wertekanons möglich. Dieser findet sich in der gemeinsam geteilten bürgerlichen Kultur wieder. Bürgerliche Kultur war nicht einfach ein Korrelat spezifischer Erwerbs- und Berufslagen, vielmehr stellte sie eine besondere und selbstständige Sphäre dar. Bürgerlichkeit als Kultur gab demgegenüber lediglich die Richtung vor, stellte ein Regelsystem dar, an welchem individuelles Handeln sich orientieren konnte. Nun erreichte diese Art der kulturellen Vergesellschaftung jedoch nie alle Gruppen im gleichen Maße und blieb damit stets auch eine Utopie; ${ }^{8}$ sie ließe sich also eher begreifen als „ein idealtypisches System von Werten und Praktiken, welches zwischen den unterschiedlichen Lebensordnungen der Welt vermittelt“. ${ }^{9}$ In Anlehnung an den Sozialhistoriker Jürgen Kocka lassen sich nun zusammenfassend folgende zentralen Merkmale bürgerlicher Kultur herausstellen: ${ }^{10}$ So zeichnet sie sich etwa a) durch eine besondere Hochachtung individueller Leistungen und damit verknüpft, einer grundsätzlich positiven Einstellung gegenüber regelmäßiger (Erwerbs-)Arbeit aus; b) weiterhin hält die bürgerliche Kultur zu einer rationalen und methodischen Lebensführung an, zur Kontrolle der Emotionen und zur Disziplin; gleichwohl fördert sie c) das Streben nach selbständiger Gestaltung individueller und gemeinsamer Aufgaben - solches spiegelt sich auch in den typisch bürgerlichen Organisationsformen wider, etwa dem bürgerlichen Vereinswesen; d) so weist bürgerliche Kultur eine hohe Affinität und Wertschätzung vor allem des Anspruchs nach selbstständiger Bildung auf; e) in diesem Rahmen der Bildungskultur zeichnet sich Bürgerlichkeit insbesondere durch ein ästhetisches Verhältnis zur Hochkultur aus, ebenso wie durch einen hohen Respekt gegenüber den Wissenschaften; f) es wird das Fundament für die bürgerliche Lebensführung im Bürgertum schon in der familiären Sozialisation gelegt, basierend auf einem dem Bürgertum eigentümlichen Familienideal, in dem

Vielfalt Europas, Göttingen 1987, S. 9-75, hier S. 10; M. RAINER LEPSIUS, Zur Soziologie des Bürgertums und der Bürgerlichkeit, in: Jürgen Kocka (Hg.): Bürger und Bürgerlichkeit im 19. Jahrhundert, Göttingen 1987, S. 79-100, hier S. 86; M. RAINER LePsiUs, Das Bildungsbürgertum als ständische Vergesellschaftung, in: Ders. (Hg.), Bildungsbürgertum im 19. Jahrhundert, Teil 3: Lebensführung und ständische Vergesellschaftung, Stuttgart 1992, S. 8 18, hier S. 8.

8 Vgl. Friedrich H. TenBruck, Bürgerliche Kultur, in: Friedhelm Neidhardt/M. Rainer Lepsius/Johannes Weiß (Hg.), Kultur und Gesellschaft (Kölner Zeitschrift für Soziologie und Sozialpsychologie, Sonderheft 27), Köln 1986, S. 263-285, hier S. 272.

9 MANFRED HETTLING, Bürgerliche Kultur - Bürgerlichkeit als kulturelles System, in: Peter Lundgreen (Hg.), Sozial- und Kulturgeschichte des Bürgertums, Göttingen 2000, S. 319-339, hier S. 322.

10 Vgl. KockA, Das europäische Muster und der deutsche Fall (wie Anm. 7), S. 18-23. 
die Familie als emotionaler Ort der Entlastung gegenüber einem zunehmend rationalisierten Alltag, als geschützter Innenraum der ,Privatheit' in strenger Abgrenzung zur, Öffentlichkeit` wirkte (und mitunter noch immer wirkt); g) letztendlich war Bürgerlichkeit zunächst und in erster Linie ein urbanes Phänomen.

\section{Die ,Intelligenz‘ und das ,bürgerliche Erbe` im Staatssozialismus}

Während die Reste eines durch Kriegseinwirkungen ohnehin geschwächten Wirtschafts- und Unternehmerbürgertums durch die Bodenreform 1945 und die im Jahre 1946 folgende erste Enteignungswelle im Industrie- und Handwerksbereich, einem Volksentscheid zur Enteignung der Kriegsverbrecher folgend, nahezu aufgerieben wurden, ${ }^{11}$ war die Situation für das Bildungsbürgertum, und damit einen Teil der Intelligenz, eine grundsätzlich andere. Gegenüber der alten, bürgerlichen Intelligenz war man durchaus zu Konzessionen bereit, brauchte man sie doch als ,Bündnispartner' zum Wiederaufbau des vom Kriege gezeichneten Landes, sowie als Vermittler und Ausbilder für spätere Generationen der neuen, sozialistischen Intelligenz. Zahlenmäßig war die Schicht dieser oftmals dem „Herrschaftssystem nur existenziell zugewandten, alten Intelligenz" 12 in der SBZ/DDR durch Kriegseinwirkung und Weggang, insbesondere in die westlichen Besatzungszonen, stark geschrumpft. Auch politisch gab es keine einheitlichen Positionen innerhalb der Intelligenzschicht: sie reichten von konservativ bis marxistisch. Dennoch war der ideologisch-politische Druck nicht auf alle Berufsgruppen gleich. Ausschlaggebend waren der planwirtschaftliche Stellenwert der jeweiligen Berufe und die Möglichkeiten, alte, vorwiegend bürgerliche Berufsinhaber durch neue Funktionsträger zu ersetzen. ${ }^{13}$ Anfangs noch konnten Angehörige der oft bürgerlich sozialisierten Intelligenz, unter Wahrung verschiedener Privilegien, führende Positionen, mit oder auch ohne Zugeständnisse an Ideologie und Politik, einnehmen und „den Staat mit außergewöhnlichen Forderungen fast erpressen, ihn nach außen repräsentieren helfen, zurückgezogen ordentliche Arbeit leisten und eine abwartende, kritisierende oder ablehnende Haltung einnehmen, die dann bei bedrohlichen Anlässen auch zum Verlassen der DDR führen konnte“. ${ }^{14}$

11 Vgl. Thomas Grossbölting, Bürgertum, Bürgerlichkeit und Entbürgerlichung in der DDR, in: Aus Politik und Zeitgeschichte. Beilage zur Wochenzeitung Das Parlament 2008, Nr. 9-10, S. 17-25, hier S. 21.

12 Guntolf Herzberg, Anpassung und Aufbegehren. Die Intellektuellen der DDR in den Krisenjahren 1956/58, Berlin 2006, S. 36.

13 Vgl. ARnd BAUerKäMPER, Kaderdiktatur und Kadergesellschaft. Politische Herrschaft, Milieubindung und Wertetraditionalismus im Elitenwechsel in der SZB/DDR von 1945 bis zu den sechziger Jahren, in: Peter Hübner (Hg.), Beiträge zur Sozialgeschichte der DDR, Köln/Weimar/Wien 1999, S. 37-65, hier S. 38; GROssBÖLTING, Bürgertum, Bürgerlichkeit und Entbürgerlichung (wie Anm. 11), S. 22.

14 Herzberg, Anpassung und Aufbegehren (wie Anm. 12), S. 42. 
So sehr man den Repräsentanten des alten Bürgertums zusetzte, so attraktiv blieben aber traditionelle Leitbilder. Anna-Sabine Ernst verweist diesbezüglich auf eine Paradoxie, denn gerade „bürgerliche Werte und Verhaltensnormen waren das Modell, an dem die Partei- und Staatsführung sich bei der Konzeption der sozialistischen Leitbilder orientierte“. ${ }^{15}$ Dem fügt sie hinzu: „Das kulturelle Erbe eignete sich nun in besonderer Weise als ideologische Klammer, gewissermaßen als kleinster gemeinsamer Nenner für die Gewinnung der bürgerlichen Intelligenz. Die angestrebte Vollendung der bürgerlichen Revolution und auch die wesentlich daraus folgende Favorisierung des klassischen kulturellen Erbes kann nicht allein als taktisch-bündnispolitische Zweckmäßigkeit interpretiert werden."16 Die Versuche der späten 1940er- und frühen 1950er-Jahre, einen ,Proletkult' ${ }^{`}$ "u etablieren, scheiterten ebenso wie der ,Bitterfelder Weg' der 60er-Jahre. „Stattdessen knüpfte man in vielfältiger Weise an die Traditionsbestände des 19. und frühen 20. Jahrhunderts an. Die Goldschnitt-Ausgabe der Werke deutscher Klassiker, die tief in der bürgerlichen Tradition verwurzelte Theaterkultur der frühen DDR, die demonstrative Bildungsbeflissenheit hoher SED-Funktionäre - in vielfacher Hinsicht schlug das Erbe der deutschen Arbeiterbewegung in Kaiserreich und Weimarer Republik durch, die ihrerseits deutliche Anleihen beim bildungsbürgerlichen Habitus und Kanon gemacht hatte. "17 Dies ließe sich unter anderem auch in Umgangsformen beobachten: etwa wenn man recht schnell vom proletarischen „Du“ zum bürgerlichen „Sie“ zurückkehrte.18 Bürgerliche Kultur blieb also anscheinend, Leitkultur'.

Im Osten Deutschlands gab es also, dies wurde bereits angedeutet, durchaus bildungsbürgerliche Kontinuitäten. Allerdings blieb bildungsbürgerliche Kultur zumeist „subkutan“ 19 und damit subkulturell. Betrachtet man diese Kulturform in der DDR als eigenständige Größe, so Günter Wirth - und hierbei betont auch er die Möglichkeiten zur Eigen- oder ,Selbstständigkeit' als konstitutiv für bürgerliche Kulturpraktiken -, so geschehe es um den Preis des Verzichts auf gesamtgesellschaftliche Effizienz: „Es bedeutet dies, dass die relativ eigenständige bildungsbürgerliche Kultur - je nach übergreifenden gesamtgesellschaftlichen Konjunkturen mehr oder weniger - zurückgedrängt wurde ins Private, Berufliche und Halböffentliche. "20 In diesen Nischen konnte sie auf zum Teil eigentümliche Weise kondensieren und sich auch konservieren, wobei dieser Handlungs- und Sozialisationsrahmen kurioserweise weniger auf zeitgenössische Modelle als auf

15 AnNA-SABINe ERnST, Erbe und Hypothek; (Alltags-)kulturelle Leitbilder in der SZB/DDR 1945-1961, in: Kultur und Kulturträger in der DDR: Analysen, hrsg. von der Stiftung Mitteldeutscher Kulturrat, Berlin 1993, S. 9-72, hier S. 13.

16 Ebd.

17 GrossBÖlting, Bürgertum, Bürgerlichkeit und Entbürgerlichung (wie Anm. 11), S. 23.

18 Vgl. ebd.

19 GÜNTER WIRTH, Zu Potsdam und anderswo. Kontinuitäten des Bildungsbürgertums in der DDR, in: Manfred Hettling/Bernd Ulrich (Hg.), Bürgertum nach 1945, Hamburg 2005, S. 85-110, hier S. 100.

20 Ebd. 
Leitmodi des 19. Jahrhunderts orientierte. ${ }^{21}$ So konstatiert der Kulturwissenschaftler Paul Kaiser vier Sozialräume, in die „Träger und Phänomene von Bürgerlichkeit im ostdeutschen Staatssozialismus" in der SBZ und frühen DDR emigrieren konnten: Die verbliebenen Gestaltungsräume bürgerlicher Professionen, die Institutionen der künstlerischen Hochkultur, alternative Milieus, die vor allem in den Kirchen (hier vorrangig die evangelischen) entstehen konnten; schließlich in die Welt(-en) des ,Privaten'.22

Soweit bürgerliche Leistungsträger Sonderrechte genossen hatten, wurden diese sukzessive nach 1961 zurückgenommen. Der Wegfall einerseits der Aussicht auf berufliche Alternativen im Westen Deutschlands durch den Grenzschluss und das Ende von „Duldungsstrukturen“ andererseits führte zur allmählichen Auflösung bürgerlicher Gegenkulturen spätestens in den 1960er-Jahren ${ }^{23}$ mit dem „(Rest-)Bildungsbürgertum“ als deren wesentliche Trägergruppe. Im Gegensatz dazu war die „Revitalisierung bürgerlicher Sozialitäts- und Vereinigungsformen“ ab den 1970er-Jahren nicht mehr allein an diese Milieus gebunden. ${ }^{24}$ Mit dem Ausweichen in das ,Private oder ,Berufliche“ wurden Chancen der „Bewahrung und Bewährung des Bürgerlichen“25 ausgelotet, nach Möglichkeit - nicht zwingend im Dialog mit anderen. Es waren einzelne Personen, keine Schichten mehr, die hier Bildungsbürgerliches repräsentierten. ${ }^{26}$

Zwei zentrale Merkmale von Bürgerlichkeit waren die Möglichkeit zur selbstbestimmten Organisation, etwa in Vereinsform, sowie eine existierende pluralistische Öffentlichkeit. Beides wurde in der DDR nur bedingt ermöglicht. Ein öffentlicher Diskurs im eigentlichen Sinne existierte nicht, die Selbstorganisation ihrerseits war stark eingeschränkt. An die Stelle der ,bürgerlichen Öffentlichkeit ${ }^{\star}$ traten also Substitute in Form von Organisationen und Ritualen. ${ }^{27}$ Gleichwohl existierten offizielle, ,vereinsadäquate Institutionen, in denen eine begrenzte Selbstorganisation und Selbstverwaltung möglich war.

21 Paul KaISeR, Die „Erfurter Ateliergemeinschaft“ - ein bürgerlicher Kunstverein im DDR-Staatssozialismus?, in: Karl-Siegbert Rehberg/Paul Kaiser (Hg.), Abstraktion im Staatssozialismus. Feindsetzungen und Freiräume im Kunstsystem der DDR, Weimar 2003, S. 149-175, hier S. 155.

22 Vgl. ebd., S. 154.

23 KAISER, Die „Erfurter Ateliergemeinschaft“" (wie Anm. 21), S. 160 f.

24 Vgl. ebd., S. 165.

25 Wirth, Zu Potsdam und anderswo (wie Anm. 19), S. 102.

26 Vgl. ebd.

27 Vgl. Thomas Grossbölting, Entbürgerlichte die DDR? Sozialer Bruch und kultureller Wandel in der ostdeutschen Gesellschaft, in: Manfred Hettling/Bernd Ulrich (Hg.), Bürgertum nach 1945, Hamburg 2005, S. 407-432, hier S. 419; KAISER, Die „Erfurter Ateliergemeinschaft" (wie Anm. 21), S. 158. 


\section{Die Klubs der Dresdner Intelligenz und ibre Protagonisten}

Insgesamt ist von einer ganzen Reihe von Klubs und Vereinigungen auszugehen, in denen sich die Dresdner Intelligenz organisierte, ${ }^{28}$ fast ausschließlich unter dem Dach des Kulturbundes. Tatsächlich wurde der erste derartige „Klub der Kulturschaffenden“, wie der spätere Klub der Intelligenz „Victor Klemperer“ zu Beginn genannt wurde, bereits im April 1953 eröffnet. ${ }^{29}$ Dem Klubvorstand gehörten an: der Arzt Albert Fromme, der damalige Dresdner Oberbürgermeister Walter Weidauer, der Generalintendant Karl Görs, die Malerin Lea Grundig, der Direktor des Deutschen Hygienemuseums Walter Friedeberger, die Schriftstellerin WeighardtLazar, der Direktor des Dresdner Zoos Wolfgang Ullrich, der Komponist Johannes Paul Thilmann und schließlich der Leiter des Instituts für Denkmalpflege Hans Nadler. Überblickt man die gesellschaftlichen Positionen dieser Dresdner Persönlichkeiten, so ist zu bemerken, dass hier ein breites Spektrum versammelt war, das Wissenschaft, Kunst und Kultur umfasste. Sein erstes Domizil fand der Klub im Dresdner Stadtteil Weißer Hirsch, Bergbahnstraße 12. Späterhin wurde ein Gebäude, gelegen in der August-Bebel-Straße 12, bezogen (das heutige „Tusculum“), das bis zur Vereinigung mit dem „Dresdner Klub“ 1972 Klubhaus bleiben sollte und dessen Rechtsträger der Deutsche Kulturbund war. ${ }^{30}$ Dem geistigen und kulturellen Anspruch der Dresdner Intelligenz, der zumeist noch aus der Tradition dieser alten Kultur- und Bürgerstadt herrührte, sollte auch die Programmplanung gerecht werden: Die Künste standen im Mittelpunkt, ebenso die Naturund Geisteswissenschaften, wie man den Angaben der Zeitzeugen und den vorhandenen Unterlagen entnehmen kann.

Neben diesen „Klub der Kulturschaffenden“ trat im Frühjahr 1957 in Dresden ein zweiter Intelligenzklub, der in seinem Anspruch gänzlich anders konzipiert und hinsichtlich seiner Ausstattung wohl einmalig war.

28 Erwähnt wurden als in Dresden ansässig bereits der „Semper-Club“ sowie Ableger der „Pirckheimer-“ und der „Goethegesellschaft“. Die Anführung beruht nicht auf Vollständigkeit. Hier empfiehlt sich weitergehende Forschungsarbeit. Aktivitäten sind vor allem ab den späten 1970er-Jahren zu verzeichnen; vgl. Sächsisches Staatsarchiv - Hauptstaatsarchiv Dresden (im Folgenden: HStA Dresden), 12485 Kulturbund der DDR, Bezirksleitung Dresden.

29 Vgl. Informationsblatt zur Eröffnung des Klubs, hrsg. vom „Klub der Kulturschaffenden“, Stadtarchiv Dresden (im Folgenden: StA Dresden), 13.50/12.

$30 \mathrm{Vgl}$. Zuarbeit zur Vorlage über die Zusammenführung des Klubs der Intelligenz Victor Klemperer und des Dresdner Klubs: zur Charakteristik des Klubs der Intelligenz „Victor Klemperer“, 5 Seiten (StA Dresden 13.50/Nr. 16). 


\section{Der „Dresdner Klub“31}

Offiziell wurde der Klub am 23. März 1957 in den Räumlichkeiten des LingnerSchlosses eröffnet. Überliefert ist die Eröffnungsrede des Ersten Vorsitzenden des Dresdner Klubs, Manfred von Ardenne. ${ }^{32}$ In dieser äußerte er sich über die Intentionen, die mit der Gründung eines zweiten Intelligenz-Klubs in Verbindung standen, bringt hier die Regierung selbst als Ideengeber ins Spiel, erwähnt Walter Ulbricht jedoch nicht explizit. Auf diesen persönlich berief er sich noch 1955 in einer Stellungnahme gegenüber dem Dresdner Oberbürgermeister Walter Weidauer, in der er wirbt: Damit der schöne Gedanke des stellvertretenden Ministerpräsidenten Ulbricht, einen Klub der Dresdner Intelligenz im „Lingner-Schloß“ ins Leben zu rufen, ein voller Erfolg wird, ist folgendes notwendig: Der Klub muss nicht nur durch die schöne Lage seines Klubhauses, sondern durch eine Reibe von Attraktionen so organisiert sein, daß die Dresdner Intelligenz ibn mit ibren Familien gern und regelmäßig besucht. ${ }^{33}$ Daraus zieht von Ardenne den Schluss, dass der Klub besonders mit solchen Eigenschaften und Einrichtungen ausgestattet sein muß, die der Einzelfamilie in der eigenen Wobnung nicht zur Verfügung stehen ${ }^{34}$ und schlägt im Anschluss daran sogleich die Einrichtung einiger Annehmlichkeiten vor: einer Terrasse mit Stuhlgruppen, einen Speiseraum, ein Tanzzimmer, einen Unterhaltungsraum mit Sesselgruppen, ein Kaminzimmer mit großen Sofas, ein Bibliothekszimmer mit Zeitschriften (auch ausländischen), ein Musikzimmer (mit Flügel und Schallplattenarchiv), sodann auch ein Fernsehzimmer, ein Billardzimmer und einen Wintergarten. Für die Außenanlage waren ursprünglich ein Parkplatz, ein Tennisplatz, ein Schwimmbassin und bequeme Bänke vorgesehen, auch die Möglichkeit einer Bootsanlegestelle und die Einrichtung von Stallungen für zwei Reitpferde sollten geprüft werden. ${ }^{35}$ Vieles davon konnte in den Jahren des Umbaus 1956/57 unter der Leitung des Architekten Gerhard Guder umgesetzt werden; ein Schwimmbassin etwa oder klubeigene Reitpferde hat es jedoch nie gegeben. Das Vorhaben war ehrgeizig: Die noch 1955 veranschlagte Planungssumme von $812.900 \mathrm{DM}^{36}$ musste im Folgejahr aufgestockt werden, sodass am Ende des Umbauprojektes die Kosten auf insgesamt 1.578.500 DM beträchtlich angewachsen waren. ${ }^{37}$

31 Über den „Dresdner Klub“ erschien bereits eine verkürzte Abhandlung; vgl. Michael Kunz/KarSTEN Klein, Bürgerlichkeit und ,Sozialistische Intelligenz'? - zum ,Dresdner Klub‘ von 1957 bis 1972, in: Dresdner Hefte 93 (2008), S. 83-89.

32 Vgl. MANFRED vON ARdenNE, Gedanken über Zweck und Bedeutung des „Dresdner Klubs“. Zur Eröffnung des Klubs im ehem. Lingner-Schloss am 23.03.1957, S. 1 (StA Dresden 13.50/17).

33 Brief von Ardenne an Oberbürgermeister Weidauer vom 27. Juni 1955 (StA Dresden 13.50/34, Bd. 2/2).

34 Ebd.

35 Ebd.

$36 \mathrm{Vgl}$. Brief von Staatssekretär Geyer an Jahn vom 17. August 1955 (StA Dresden 13.50/34, Bd. 2/2).

37 Vgl. Kostenaufschlüsselung (StA Dresden 13.50/34, Bd. 2/2). 
Vieles spricht nun allerdings dafür, dass die Anregung von Seiten von Ardennes selbst gekommen war; dass die Gründung eines entsprechenden Klubs darüber hinaus von ihm intensiv betrieben wurde, wie auch Zeitzeugen bestätigten. Bereits einen Tag nach seiner Ankunft in Dresden im Frühjahr 1955 empfing von Ardenne, wie seinen Memoiren zu entnehmen ist, Walter Ulbricht in seinem Anwesen auf dem Weißen Hirsch. ${ }^{38}$ Wahrscheinlich ist also, dass von Ardenne bei dieser Begegnung neben der Klärung der Forschungsarbeit seines Instituts auch die Gründung eines weiteren Intelligenz-Klubs anregte.

Eigentümlich sind die Zuständigkeiten für den „Dresdner Klub“: Nicht, wie sonst üblich, war er dem Kulturbund unterstellt, sondern er blieb eine Einrichtung der Stadt Dresden, deren Oberleitung er auch offiziell unterstand. ${ }^{39}$ Die eigentlichen Impulse, die mit der Gründung des „Dresdner Klubs“ in Verbindung standen, lassen sich einem Dossier entnehmen, das um 1971 im Zuge der geplanten Zusammenlegung mit dem Klub der Intelligenz „Victor Klemperer“ für die SEDBezirksleitung angefertigt wurde, nämlich dem Zusammenschluss der beim Aufbau des Sozialismus in der DDR schöpferisch tätigen Persönlichkeiten auf wissenschaftlichem, künstlerischem und politischem Gebiet mit dem Ziel gegenseitigen Gedankenaustausches und geistiger Entspannung zu dienen, [...] die gesellschaftlichen Bindungen der Mitglieder untereinander [zu] festigen, besonders jener, die nach erfolgreicher wissenschaftlicher Arbeit in der SU in die DDR kamen, [sowie] durch kollektive Formen der Geselligkeit und Freizeitgestaltung individualistischer Isolierung entgegenwirken. ${ }^{40}$ Offensichtlich versuchte man also, den um 1955 aus der Sowjetunion zurückkehrenden Wissenschaftlern einen gesellschaftlichen Anschluss zur Intelligenz vor Ort zu vermitteln. Ebenso bestand wohl das Bestreben - zumindest schien es in der Weise intendiert -, dass diese Rückkehrer eine Vermittlerrolle zwischen der Dresdner Intelligenz, deren Vertreter noch überwiegend dem alten Bildungsbürgertum zuzurechnen waren, und dem lokalen Führungspersonal der SED übernehmen und damit jener, individualistischen Isolierung' entgegenwirken. Dies war wohl nur im begrenzten Maße erfolgreich.

Von Ardenne indessen hatte eigene Vorstellungen, wenn er explizit vor allem das direkte Gespräch zu zweit oder zu dritt, möglichst in völlig zwanglosem Rabmen, [das] in steigendem Maße die wabre Quelle wichtigster Anregungen für den Fortschritt in Wissenschaft, Technik und Industrie, sowie wobl auch in der Kunst [wird], einforderte. ${ }^{41}$ Damit war zugleich der Rahmen abgesteckt, in dem der geistige und fachliche Austausch stattfinden sollte. Das ,zwanglose Gespräch“ ist hier auch als ein Idealbild bildungsbürgerlichen Konversationsanspruchs zu verstehen. Ebenfalls in der Eröffnungsrede findet sich der Hinweis auf eine, nach seiner Mei-

38 Vgl. Manfred von Ardenne, Die Erinnerungen, München 1990, S. 265.

39 Zuarbeit der Bezirksleitung der SED, Abteilung Kultur, über die Problematik der Zusammenlegung des Dresdner Klubs und des Dresdner Klubs der Intelligenz „Victor Klemperer" des Deutschen Kulturbundes, S. 6 (StA Dresden 13.50/16).

40 Ebd., S. 2.

41 Von Ardenne, Gedanken (wie Anm. 32), S. 7. 
nung, steigende Beanspruchung der Geistesarbeiter, die ja zur Hebung des allgemeinen Lebensstandards ihre Kräfte bis zur physischen Grenze einsetzten. Er spricht hier sogar von einer Zunahme der sogenannten Manager-Krankheit gerade bei den geistigen Führungskräften. [...] Diesem Kreis hoch beanspruchter Geistesarbeiter wollte die Fürsorge unserer Regierung eine neue, eine einzigartige Möglichkeit für wirkliche und häufige Entspannung und Erholung erschließen. ${ }^{42} \mathrm{Im}$ Kern wurde die Forderung gestellt, einer Unabhängigkeit und Freizügigkeit in der Meinungsäußerung und einer quasi ins Private verlegten Konversation zuzustimmen, beziehungsweise diese als erforderlich angemahnt; politische Einflussnahme seitens der SED-Führung wurde damit weitestgehend vermieden. Im Gegenzug und sozusagen als Preis für die gewährte Freiheit bot von Ardenne nun fachliche Leistungen, die bei Gewährung solcher Privilegien sich ökonomisch und kulturell zum Wohle der Allgemeinheit niederschlagen sollten. Entsprechend dieser Zielsetzung benannte er sehr konkret jenen Personenkreis, aus dem sich die Angehörigen des Klubs rekrutieren sollten - die vorgesehene Gewinnung nur ausgesuchter Mitglieder begründet er darwinistisch: Eines der Grundelemente, das seit den Tagen der ersten biophysikalischen Schöpfung lebender Substanz den Entwicklungsfortschritt lebendiger Wesen bestimmt, ist das,Auswablprinzip' [...]. Ein äbnliches Auswahlprinzip steuert die Zusammensetzung der geistigen Fübrungsschicht in der sozialistischen Gesellschaft. Nicht das Geld und die Stellung der Eltern, nicht die Herkunft, sondern die persönliche Leistung [...] sind bei ibr [...] entscheidend für den persönlichen Lebenserfolg. [...] Menschen aus diesem Kreis, und nur aus diesem Kreis, sind es, die wir als Mitglieder unseres Klubs uns wünschen und sammeln wollen. ${ }^{43}$ Damit kam recht klar zum Ausdruck, welcher gesellschaftliche Kreis vorzüglich - und selektiv - angesprochen werden sollte. Ganz klar wurde in diesem Passus auf das einende Moment der Bildung rekurriert, der Bezug zur ,persönlichen schöpferischen Leistung' herausgestellt. Unüberhörbar forderte von Ardenne dies auch von den potenziellen Mitgliedern des neuen Klubs. Eine statistische Auswertung der Mitgliederliste des „Dresdner Klubs“44 untermauert diesen Anspruch:

42 Ebd., S. 1.

43 Von Ardenne, Gedanken (wie Anm. 32), S. 3.

44 Es handelt sich hierbei um 51 ursprünglich maschinenbeschriebene, lose Seiten, die durch Einlageblätter in Anfangsbuchstaben der Nachnamen unterteilt sind. Zahlreiche nachträgliche maschinelle und handschriftliche Zusätze und Streichungen wurden vorgenommen, die darauf hindeuten, dass die Liste als offizielles Mitgliederverzeichnis geführt wurde (StA Dresden 13.50/17). 
Unterteilt nach Professionen

nominal prozentual

Wissenschaftler, Hochschullehrer (exkl. Medizin und Kunst):

91

$27,2 \%$

Ingenieure, Techniker, Ökonomen, Architekten u. ä.:

Mediziner (inkl. Veterinärmediziner):

$16,8 \%$

hiervon:

Habilitation vermerkt:

19

Professur (Medizin, auch emeritiert):

Künstler/Kulturschaffende:

20

staatl. Institutionen/Parteikader/Militär:

56

83

$24,9 \%$

Redakteure:

Juristen:

sonstige $45 /$ nicht angegeben:

\begin{tabular}{cc}
44 & $13,2 \%$ \\
10 & $2,9 \%$ \\
6 & $1,8 \%$ \\
1 & $0,3 \%$ \\
44 & $13,2 \%$ \\
\hline
\end{tabular}

Tabelle 1: Nominale und prozentuale Verteilung der Professionen im Mitgliederkreis des „Dresdner Klubs“.

Der Kreis der Mitglieder war, ganz nach dem Geschmack von Ardennes, fachlich durchaus heterogen aufgestellt. Dazu gehörten neben ,kulturellen' Größen wie Karl von Appen, Theo Adam, Rudolf Neuhaus, Lea Grundig, Karl Laux, KarlFriedrich Fuchs, Kurt Masur und Max Seydewitz auch die wissenschaftlich-technische Elite Dresdens, von Gerhard Potthoff über Klaus Fuchs und Heinz Pose bis zu Béla Barényi und Gerhard Rehbein. Auffallend auch der relativ hohe Anteil an Medizinern; hier versammelt insbesondere Klinik-Direktoren, Oberärzte und Medizinalräte: Helmut Born, Gottfried Dominok, Ernst Marré oder Heinrich Fritz. Kein Widerspruch war auch die Mitgliedschaft hoher Funktionäre, des Ersten Sekretärs der Bezirksleitung der SED Oswin Forker etwa, des Dresdner Oberbürgermeisters Walter Weidauer und des Ersten Sekretärs der Stadtleitung der SED Max Renne. Die Aufzählung ist exemplarisch, sollte aber verdeutlichen, welche Ausmaße die geplante Vernetzung der Dresdner Fachkapazitäten annahm. Die folgende Tabelle verweist auf die Verteilung von akademischen Graden im „Dresdner Klub“:

\begin{tabular}{lrr} 
Höchster akad. Grad: & nominal & prozentual \\
\hline Professor: & 112 & $33,5 \%$ \\
Doktor: & 90 & $26,9 \%$ \\
Diplom: & 51 & $15,3 \%$ \\
ohne erkennbaren Grad: & 81 & $24,3 \%$ \\
\hline
\end{tabular}

Tabelle 2: Nominale und prozentuale Verteilung akademischer Titel bei den Mitgliedern des „Dresdner Klubs" (Prozentsatz auf eine Stelle nach dem Komma gerundet).

45 Die Kategorie „sonstige“ umfasst jene Fälle, bei denen eine eindeutige Zuordnung nicht möglich, bzw. die berufliche Tätigkeit nicht vermerkt ist. Hierzu zählen unter anderem die als „Witwen“ geführten Personen. Weiterhin lassen sich die verschiedenen geführten akademischen Grade der Mitglieder und ihre prozentuale Verteilung eruieren. 
Die Anzahl der Mitglieder dürfte sich im Verlauf des Bestehens des Klubs zwischen 300 und etwa 360 bewegt haben. Einer Sozialstatistik aus dem Jahre 1971 zufolge 46 wurden für den „Dresdner Klub“ folgende Zahlen ermittelt: 1970: 356; 1971: 338 .

Nachfolgende Darstellung ${ }^{47}$ soll die ,leitenden Positionen' abbilden, welche von verschiedenen Mitgliedern des „Dresdner Klubs“ besetzt wurden, und im hier möglichen Umfang erfassen:

\section{Personen in leitender Position}

\begin{tabular}{lr} 
Direktor allgemein ${ }^{48}$ (außer Generalmusikdirektor): & 45 \\
Generalmusikdirektor: & 3 \\
Rektor/Dekan: & 5 \\
Generalintendant/Intendant: & 2 \\
Chefarzt/Oberarzt: & 22 \\
Chefingenieur/Oberingenieur: & 7 \\
Chefarchitekt: & 2 \\
beratender Ingenieur: & 2 \\
Parteisekretär der SED-Bezirksleitung: & 1 \\
Oberbürgermeister (auch im Ruhestand): & 2 \\
\hline
\end{tabular}

Tabelle 3: Nominale Verteilung von, leitenden Positionen` in Beruf und Kultur, die von Mitgliedern des „Dresdner Klubs“ ausgeübt wurden.

Was das kulturelle Programm betrifft, so scheint es sich tatsächlich um ein klubintern gestaltetes zu handeln. Von Beginn an ${ }^{49}$ wurde den Mitgliedern ein breites Angebot an verschiedensten Veranstaltungen offeriert: Dies reichte von wissenschaftlichen Vorträgen, über gesellige Tanzveranstaltungen bis hin zu Filmvorfüh-

\footnotetext{
46 Vgl. Dossier „Angaben über die Mitgliederentwicklung des Dresdner Klubs“ (StA Dresden 13.50/16).

47 Die Kategorien sind keinesfalls erschöpfend, schon allein in Ermangelung einer akzeptablen Definition von ,leitender Position'. Anhaltspunkt sollen ,herausgehoben' zu nennende Berufspositionen sein, die sich durch Zusätze wie „Ober-“ oder „Chef-“ oder durch andere Prädikate von den übrigen hierarchisch absetzen. Auch sind natürlich personelle Überschneidungen zu berücksichtigen, sodass die Zahlen nicht als absolut betrachtet werden dürfen; ein Beispiel soll dies verdeutlichen: So ist etwa der Humanmediziner Prof. Dr. med. habil. Joachim Holtorff sowohl „Erster Oberarzt“ als auch „Ärztlicher Direktor der Medizinischen Akademie Dresden“. Zudem begleiten einige der Klub-Mitglieder zusätzliche Ämter und Würden, wie Ehrenmitgliedschaften, die im Einzelnen hier nicht abgebildet und daher nur exemplarisch aufgegriffen werden können. Einen solchen Fall stellt etwa Prof. em. Dr. h. c. Ernst-Herrmann Meyer dar: Wohnhaft in Berlin, war er Komponist und Musiktheoretiker, zugleich auch Vizepräsident der Deutschen Akademie der Künste Berlin sowie Nationalpreisträger und Professor.

48 Hierunter erfasst werden sämtliche berufliche Positionen, die in der Mitgliederliste ausdrücklich mit der Bezeichnung oder dem Zusatz „Direktor“ geführt werden. Die einzelnen Tätigkeiten können hierbei stark variieren (Instituts- und Werksdirektor, Technischer Direktor, Zoodirektor, Generaldirektor der Staatlichen Kunstsammlungen etc.).

49 Erhalten ist unter anderem das erste Programmheft aus dem Jahre 1957: „Der Dresdner Klub - Veranstaltungen, Vorankündigungen“" (StA Dresden 13.50/16).
} 
rungen. Bemerkenswert ist hier die Themenvielfalt. Sie richtete sich an ein Publikum mit entsprechender Vor- bzw. breit angelegter Bildung. Dies würde im Wesentlichen der Konzeption, die von Ardenne für den Klub vorgeschlagen hatte, entsprechen. Darüber hinaus gab es regelmäßige und unregelmäßige Veranstaltungsreihen, Geselligkeiten und verschiedene Zirkel. Auch hier nur eine Auswahl: Tanzzirkel, Damennachmittag, Modenschauen, Klub- und Spielabende, verschiedene Feste: Karneval, das „Rosenfest“ auf der Terrasse mit Tanzkapelle, Kostümbälle, „Großes Hausschlachtfest“, „Fröhliches Winzerfest“, Liederabende, ein Sprachkurs „Brush up your English“ sowie Gemeinschaftsveranstaltungen mit dem „Freundeskreis der Gemäldegalerie Neue Meister“.

Nicht alle Veranstaltungen richteten sich freilich an sämtliche Mitglieder. So ist die Vielfalt wohl generell nur als ein Angebot zu betrachten, dem ein Angehöriger des Klubs nach individuellem Interesse folgen konnte. Gesellige Runden, aber auch Tanzzirkel oder Modenschauen sind als eine zwanglose Offerte vor allem an die Angehörigen der Klubmitglieder verstanden worden, zumal der Klub die Familien einbezog. Bei den Mitgliedern selbst zeigen sich unterschiedliche Präferenzen. Eine Reihe von Veranstaltungen widmete sich auch der gesellschaftspolitischen Entwicklung im ,nichtsozialistischen Ausland'. Dem Interesse nach weitergehenden Eindrücken kam man durch Reiseberichte, wie etwa die des OB Weidauer über eine Ägyptenreise ${ }^{50}$ oder späterhin „Japan - aus der Sicht eines Verkehrsfachmannes“, entgegen. Bereits im späten 18. und über das gesamte 19. Jahrhundert hinweg hatte es ein gesteigertes Interesse an Reise- und Milieuberichten vor allem in bürgerlichen Kreisen gegeben. Die Situation in der DDR zwang in ähnlicher Weise, sich über andere Quellen zu informieren, die eine eigene Anschauung ersetzen sollten und auch mussten. Dies waren zum einen Berichte derjenigen, welche Reisefreizügigkeit genossen, zum anderen mochten auch die reichlich im Klub gezeigten Filme aus dem ,kapitalistischen Ausland', die von den SED-Kadern moniert wurden, geeignet erscheinen, dieses Bild abzurunden. Hieraus erklären sich auch die häufigen Kinoabende.

Vor allem aber die Bibliothek und das klubeigene Restaurant erfreuten sich größter Beliebtheit und wurden als ,Attraktionen' regelmäßig und gern, so wie von Ardenne bereits im Vorfeld angedacht und wie von mehreren Zeitzeugen bestätigt, besucht. Immerhin war für die Beschaffung von Büchern ein jährlicher Etat von 4.000 DM vorgesehen. Diese Summe wird in einer Kostenauflistung aus den Jahren 1960 bis 1965 “ genannt. ${ }^{51}$

Die Veranstaltungen selbst waren wohl recht unterschiedlich frequentiert, das Lingner-Schloss nur zu ausgewählten Veranstaltungen ausgelastet. Daraus ergibt sich auch ein Teil der offiziellen Kritik an der Praxis der Abschottung in eigentlich $\mathrm{zu}$ kleinem Kreise, dass es hier unbedingt $\mathrm{zu}$ einer besseren Auslastung der Räumlichkeiten des Lingner-Schlosses kommen müsse und die staatlichen $\mathrm{Zu}$ -

$50 \mathrm{Vgl}$. ebd.

51 Vgl. „Hauptinstandsetzungen und Beschaffungen 1960-1965“ (StA Dresden 13.50/ 17, Bd. 2/2). 
schüsse auf ein Minimum zu reduzieren seien. ${ }^{52}$ Die zwischenzeitlich erfolgte zeitweise Vermietung der Räumlichkeiten, insbesondere des Restaurants, für verschiedene Veranstaltungen, traf bei einigen Klubmitgliedern auf geteiltes Verständnis.

Offensichtlich mehrten sich auch kritische Stimmen, die von zweierlei Intelligenzen in Dresden sprachen. ${ }^{53}$ Man befand, dass der „Dresdner Klub“ in seiner Anfangszeit und unter damaligen Bedingungen relativ erfolgreich arbeitete. Seit Mitte der 60er-Jahre jedoch erklärte man in der Retrospektive von 1972, dass der Klub mit seinen Mitgliedern binter den gesellschaftlichen Erfordernissen zurückblieb. ${ }^{44}$ Hauptkritikpunkte waren: fehlender Bezug zur neuen sozialistischen Intelligenz, stagnierende und sogar sinkende Mitgliederzahlen, Überalterung, das Neue und Fortschrittliche könne keinen Fuß fassen sowie jährlich sinkende Besucherzahlen bei Veranstaltungen. ${ }^{55}$ Mit Beschluss des Rates der Stadt Dresden vom 15. Juni 1972,56 dem jeweils Beschlüsse der Stadt- und Bezirksleitung der SED zugrunde lagen, wurde das Ende des „Dresdner Klubs“ politisch besiegelt. Er ging schließlich zusammen mit dem Klub der Intelligenz „Victor Klemperer“ im neuen „Dresdner Klub der Intelligenz" auf. Eine der Folgen war eine massive Austrittswelle, vor allem von Mitgliedern des ehemaligen „Dresdner Klubs“. Ein Grund war wohl, dass ihnen bei einem Verbleib im Klub die Kulturbundmitgliedschaft oktroyiert werden sollte. Zudem sahen, wie aus Stellungnahmen zur Fusion hervorgeht, viele Ehemalige die ursprünglichen Ziele des Klubs verwässert. ${ }^{57}$

\section{„Carus-Club - Dresdner Ärzte und ihre Freunde“}

Etwas anders, wenngleich nicht weniger selektiv, präsentiert sich die zweite der zu betrachtenden Institutionen: Gegründet wurde der „Carus-Club - Dresdner Ärzte und ihre Freunde im Kulturbund der DDR“, wie er vollständig hieß, am 13. Mai 1984. Ein im Vorfeld versandtes Einladungsschreiben vom 15. April, unterzeichnet in ausharrender Verehrung vom Vorsitzenden der Stadtleitung des Kulturbundes Prof. Dau sowie vom Vorsitzenden des „Carus-Clubs“ Prof. HansGeorg Lippmann, informierte über das bevorstehende Ereignis und lädt ein, eher einem Aufgebot ähnlich und in wohl so beabsichtigter, antiquierter Weise: Hoffend auf woblwollende Aufmerksamkeit wie, ein güthiges Interesse' des geneigten Lesers, sei er Arzt oder der Medizin zugewandter Naturwissenschaftler,

\footnotetext{
52 Vgl. Zuarbeit Dresdner Klub und Dresdner Klubs der Intelligenz „Victor Klemperer" (wie Anm. 39), S. 5.

53 Vorlage zur 81. Sitzung des Rates der Stadt Dresden am 15. Juni 1972 (datiert auf den 7. Juni 1972), 2 Seiten (StA Dresden 13.50/17).

54 Vgl. Dossier „Zuarbeit - Entwicklung des ,Dresdner Klubs““, S. 1 (StA Dresden $13.50 / 16)$.

55 Vgl. ebd., S. 1 f.

56 Vorlage zur 81. Sitzung des Rates der Stadt Dresden (wie Anm. 53), S. 2.

57 Vgl. diverse Einladungs- und Rückantwortschreiben mit Äußerungen zur Mitgliedschaft (StA Dresden 13.50/17).
} 
Philosoph, Künstler resp. Ingenieur, beehren wir uns, die bevorstehende Gründung eines Clubs der Dresdner Ärzte im Kulturbund der DDR, dem die Bezeichnung $C$ arus - Clu b zuteil werden soll, anzuzeigen. 58

Die einleitenden Zeilen wurden mit Zitaten des Namenspatrons Carl Gustav Carus versehen und die beiden Autoren versuchten damit einen Brückenschlag: nämlich eine Verbindung zwischen dem Wirken des Carus' seiner Zeit und dem gegenwärtigen Anliegen eines Dresdner Ärzte-Klubs aufzuzeigen und gewissermaßen an eine (so empfundene) Dresdner Tradition anzuknüpfen; noch deutlicher wird dies in der nachfolgenden Ausführung: ,Thenerste Freunde, - würde so nicht ein Billett an jene, Günstiggesinnten ..., denen die Kunst rechter und edler Lebensführung vor allem am Herzen liegt...' begonnen haben können, bätte sich Carl Gustav Carus [...], tätig in Dresden als Arzt, Naturforscher, Philosoph, Künstler vor der nämlichen Aufgabe gesehen, die uns jetzt höchst angenehmer Anlass zu diesen Zeilen ist? ${ }^{59}$ Die im Anschluss von den beiden Autoren selbst aufgeworfene Frage Warum und wozu ein Carus-Club in Dresden heute?, beantworteten diese, indem sie einen direkten Bezug zur Stadt Dresden, wiederum aber besonders zum Wirken von Carus in bürgerlich-humanistischer Tradition herzustellen versuchten: Unsere Stadt blickt auf bedeutende kulturelle und wissenschaftliche Traditionen zurück, die unter den Bedingungen des Sozialismus wie alles Progressive und Wertvolle aus der Vergangenheit nicht nur bewahrt werden, sondern in vollem Maße zur Entfaltung und Blüte gelangen. [...] In der Geschichte Dresdens verkörpert Carl Gustav Carus [...], von Goethe ob seiner Universalität schon 1818 bewundert, gleichermaßen ärztlich-humanistische Traditionen, wissenschaftliches Erkenntnisstreben, geistig-weltanschauliches Bekenntnis und breite kulturelle Interessen .60 Das Vermächtnis Carus' zu erfüllen, würde deshalb vor allem bedeuten, die großen Möglichkeiten für eine umfassende Persönlichkeitsbildung zu nutzen. Könnte sich solcher Aufgabe, ,theuerste Freunde', nicht gerade auch die medizinische Intelligenz unserer Stadt in besonderem Maße verpflichtet füblen und sich dieser stellen in der Erschließung und Pflege interdisziplinärer Begegnungen mit Persönlichkeiten der Natur-, Gesellschafts- und Technikwissenschaften, der Kunst und Kultur? In diesem Sinne will der Carus-Club wirken - seine Arbeit soll auf die Aktivierung und Wabrung geistig-kultureller Freizeit-Interessen der medizinischen und der sich ibr verwandt füblenden Intelligenz gerichtet sein." 61

Die ,offiziellen' Grundzüge der Gründung eines künftigen „Clubs der Dresdner Ärzte“ waren dann im Oktober 1983, nach mehreren vorausgegangenen Bera-

58 Kulturbund der DDR, Stadtleitung Dresden: Einladungs- und Informationsschreiben „Theuerste Freunde“ vom 15. April 1983, 2 Seiten (Archiv der Medizinischen Fakultät „Carl Gustav Carus“, Zweigstelle des Universitätsarchivs der TU Dresden - im Folgenden: Archiv der Medizinischen Fakultät - GES/02).

59 Ebd., S. 1.

60 Ebd.

61 Ebd. 
tungen auf Ebene der Bezirksleitung Dresden des Kulturbundes, ${ }^{62}$ ausformuliert. In einer von Lippmann unterzeichneten „Information zum Stand der Vorbereitungsarbeiten“ der Initiativgruppe zur Gründung des "Carl-Gustav-CarusClubs“, datiert auf den 31. Oktober 1983, ist unter anderem Folgendes angeführt: Das Ziel der Gründung dieses Clubs ist eine stärkere Mobilisierung der medizinischen Intelligenz der Stadt (und des Bezirks) Dresden für eine aktive Mitarbeit im K[ultur]B[und]. Dabei soll unter der Nutzung berufsspezifischer geistigkultureller Freizeitinteressen der med. Intelligenz die interdisziplinäre Begegnung zwischen Medizin, Wissenschaften und Künsten im Vordergrund der Clubarbeit stehen. ${ }^{63}$ So war vorgesehen, bei der Gestaltung der künftigen Klubarbeit zunächst organisierte Zusammenkünfte im Sinne interdisziplinärer Gespräche durchzuführen, aus denen heraus sich schließlich das typische Profil der künftigen Klubarbeit ergeben sollte. ${ }^{64}$ Die Initiativgruppe war bereits provisorisch organisiert bei der Stadtleitung Dresden des Kulturbundes.

Bereits ein knappes Jahr zuvor, am 18. August 1982, war es ebenfalls Prof. Lippmann gewesen, der in einem Brief an den Ersten Sekretär der Bezirksleitung Dresden des Kulturbundes der DDR, Dr. Günther Kirsch, ein „Bezirks-Aktionsprogramm“ zur "Aktivierung der Kulturbund-Arbeit mit der medizinischen Intelligenz" 65 vorstellte. Dieses wohl schon damals als Vorarbeit zur Gründung des Klubs gedachte Positionspapier bündelte die Überlegungen, welche ausschlaggebend für eine solche Assoziation hätten sein können. Unter anderem heißt es dort: Die Basis einer solchen Aktivierung und Akzentuierung sollte die Anerkennung des Vorhandenseins spezifischer geistig-kultureller Bedürfnisse der medizinischen Intelligenz [sic!] sein. Neben der Betonung des besonderen Ausmaßes an zwischenmenschlicher Kommunikation, wie es von keiner anderen Berufsgruppe erreicht wird, betont Lippmann das besonders ausgeprägte Interesse am interdisziplinären Austausch mit Nachbarwissenschaften. Im zweiten Teil des Papiers geht er sodann auf einen, nach seinem Dafürhalten besonderen Zusammenhang ein: Ärzte haben in aller Regel besonders enge Beziebungen zu den Künsten (Musik, Bildende Kunst, Darstellende Kunst), die zu einem wesentlichen Teil nicht nur den Freizeitinhalt bestimmen, sondern auch in die Arbeitssphäre bineinreichen. ${ }^{66}$ Hier will Lippmann Anknüpfungspunkte für eine Arbeit des Kulturbundes in mindestens zweierlei Hinsicht erkennen: Zum einen wären Streitgespräche über aktuelle Konzert-, Theater- und Ausstellungsereignisse mit Autoren bzw. Interpreten möglich, andererseits Gesprächsabende zu Beziehungen

62 Vgl. Kulturbund der DDR, Stadtleitung Dresden: Information zum Stand der Vorbereitungsarbeiten vom 31. Oktober 1983, 2 Seiten (Archiv der Medizinischen Fakultät GES/02).

63 Ebd., S. 1.

64 Vgl. ebd.

65 Brief von Prof. Lippmann an Dr. Kirsch: „Bezirks-Aktionsprogramm - Einige Gedanken zur Aktivierung der Kulturbund-Arbeit mit der medizinischen Intelligenz" vom 18. August 1982, S. 1 (Archiv der Medizinischen Fakultät GES/02).

66 Ebd. 
zwischen den Künsten und der Medizin, ebenfalls unter Heranziehung namhafter Künstler und Interpreten. ${ }^{67}$ In diesen Überlegungen spiegelt sich der nahezu gesamte ,bürgerliche‘ Bildungskanon wieder. Überdies postuliert Lippmann ein besonderes Verhältnis der Ärzteschaft zu den ,Künsten', bis in die ,Arbeitssphäre hinein. Wie sich dieses gestalten soll, wird an der Stelle nicht ausgeführt.

Über Struktur und Leitung finden sich Angaben in den "Satzungen“ des „Carus-Clubs“,68 zum Teil wortgleich aus den bereits zitierten Vorbereitungsschriften übernommen. Darüber hinaus werden hier Informationen zur Arbeit und Funktionsweise des Klubs gegeben. Im Absatz 3 etwa war als ein Ziel die enge Zusammenarbeit und gegenseitige Bereicherung mit anderen Einrichtungen und Vereinigungen des Kulturbundes in der Stadt Dresden, insbesondere mit dem ,Dresdner Klub der Intelligenz', dem, Gottfried-Semper-Club' und weiteren einschlägigen Interessensgemeinschaften ${ }^{69}$ formuliert. Das in der Satzung festgeschriebene Empfehlungs-Prinzip kam allerdings nicht zum Tragen. Es habe, so ein Zeitzeuge, keine großen Prämissen oder Überprüfungen gegeben; wer einen Antrag stellte, sei in der Regel aufgenommen worden. Möglicher Grund hierfür war, dass die Zahl der Bewerber überschaubar geblieben sei. Diese nun wurden zunächst vornehmlich über persönliche Ansprache rekrutiert. Die folgende Tabelle gibt Auskunft über die Häufigkeit akademischer Grade bei Mitgliedern des „Carus-Clubs“ sowie über zusätzliche Ehrentitel:

\begin{tabular}{ccccccc} 
Jahr & & \multicolumn{2}{c}{ akademischer Grad } & \multicolumn{3}{c}{ zusätzliche Ehrentitel } \\
\hline & Diplom & Doktor & Professor & MR & OMR & andere \\
1985 & 23 & 51 & 12 & 6 & 4 & 5 \\
1986 & 19 & 51 & 12 & 9 & 8 & 7 \\
1989 & 16 & 49 & 14 & 15 & 6 & 8 \\
\hline
\end{tabular}

Tabelle 4: Verteilung akademischer Grade sowie Ehrentitel bei den Mitgliedern des „Carus-Clubs“ in den Jahren 1985, 1986 und 1989.

Als akademische Grade wurden gezählt: Diplome, Doktorgrade (ohne fachliche Unterscheidung) sowie der des Professors. Als Ehrentitel werden geführt: der Medizinalrat (MR) und Obermedizinalrat (OMR). Die Kategorie „andere“ umfasst: Nationalpreisträger (NPT), Pharmazierat (PhR) und Oberpharmazierat (OPhR) sowie Sanitätsrat (SR). Weitere Titel erscheinen in den Mitgliederlisten nicht. Zu beachten gilt, dass die akademischen Grade insgesamt erhoben wurden, also nicht nur für die Hauptklientel der Ärzte. Wie zu erkennen ist, bewegte sich die Zahl der Mitglieder etwa zwischen 80 und knapp 100 Personen, die institutionellen Mitglieder nicht mit hinzugerechnet. Damit dürfte nur ein Bruchteil des

67 Vgl. ebd.

68 Vgl. „Club ,Carl Gustav Carus' des Kulturbundes der DDR - Stadtleitung Dresden - Satzungen“ (Archiv der Medizinischen Fakultät GES/02).

69 Ebd., S. 3. 
gesamten ärztlichen Personals der medizinischen Einrichtungen Dresdens hier organisiert gewesen sein.

Prof. Hans-Georg Lippmann erläuterte seine eigenen Intentionen, die zur Gründung des „Carus-Clubs“ führten, in einem Interview für die KulturbundZeitschrift „Sonntag": Den Club gründeten wir 1984, und ich darf sagen, daß die Anregungen dazu meinen Ambitionen in schöner Weise entsprachen. Ich kann mir einfach ein Leben obne Kultur und Geselligkeit nicht vorstellen, und ich weiß, daß die Begegnung mit Kunst und Wissenschaft in immer nener Sicht, was die Geschichte betrifft, in immer neuer Ausprägung, was Gegenwart und Zukunft betrifft, eine große, den einzelnen wie die Gesellschaft bereichernde Kraft darstellt. Als ich den Vorsitz übernahm, übernabm ich damit natürlich auch die Verpflichtung, jede Veranstaltung so gewissenhaft wie möglich zu bedenken und mit den anderen Mitgliedern des Vorstandes zusammen vorzubereiten. Wir wollen nicht einfach Wissen vermitteln oder einen Vortrag mit etwas Musik, umrabmen', in der Aussprache banen wir auf das Mitdenken, im musikalischen Teil auf das Ansprechen der Emotionen. Wir wollen die Begegnung, den Austausch, das Erlebnis. Das geht natürlich nicht mit Routine, man darf nicht glauben, daß sich ein gelungener Abend auf die gleiche Weise erfolgreich wiederholen läßt. Deshalb unter anderem die Sorgfalt, die wir in unsere Einladungen legen. ${ }^{70}$ Diesen Anspruch versuchte Lippmann auf den „Carus-Club“ zu übertragen.

Es widerspiegelt sich auch im Kulturprogramm, dessen Höhepunkte Dieter Meißner, der damals amtierende Vorsitzende, 1989 in einer Laudatio resümierte: Direkt medizinisch orientiert waren die Abende zur Verkehrsmedizin, zur Krebstherapie und-Prophylaxe, der von Prof. $v$. Ardenne gestaltet wurde, zur Raumfahrtmedizin, zur Musiktherapie, aber auch zur Jugend- und Altersforschung [...]. Historische Rückblicke fübrten uns mit unserem Namenspatron Carl Gustav Carus zusammen, mit Prinz Johann von Sachsen und mebrmals auch mit dem Haus Wettin. Wir begegneten dem Opernhaus, dem Hotel Bellevue und dem in Entstehung begriffenen Dresdner Hof, dem Theaterplatz - zusammen mit dem unvergessenen Fritz Löffler - der Albrechtsburg, dem Dresdner Schloß [...]. Der Musik waren mebrere Abende gewidmet [...] erinnern uns mit Freude an die Probenabende in der Semperoper, aber auch an unsere Begegnungen mit Prof. Flämig und dem Kreuzchor.71 In jenem "Sonntag“-Interview hob Lippmann auf den besonderen Charakter des Austausches, der Diskussion ab: Auch der Gehalt an Streitbarkeit will bedacht sein, was sich bei einer Problematik, die in der Praxis zum Teil experimentellen Charakter hat, von selbst ergibt. Hierher gebört, Musik in der Medizin - Musiktherapie?' Da in unseren Klub viele Ärzte kommen, die an solchen Themen interessiert sind, gibt es auch etliche unterschiedliche Meinungs-

70 „Festlich - Nichts weiter“, Interview mit Prof. Hans-Georg Lippmann, in: Sonntag Die kulturpolitische Wochenzeitung, 11. Januar 1987.

71 Ansprache des amtierenden Vorsitzenden Dieter Meißner anlässlich der 5. Wiederkehr der Gründung des Carus-Clubs vom 16. Mai 1989 (Archiv der Medizinischen Fakultät GES/02). 
äußerungen. Wir suchen die Diskussion, weil wir der Meinung sind, Diskussion bilft klären, sie führt Menschen gleichen Sinnes zusammen, sie hilft Vertrauen schaffen. Diskussion ist auch ein Appell an die Persönlichkeit, von ibrer Stärke, von ibrer individuellen Ausprägung Gebrauch zu machen. ${ }^{72}$

Ermöglicht werden sollte dies im Rahmen des Klublebens. Der Lebensweg eines Menschen werde gewöhnlich durch Neigung, Anregungen, Freunde, ein wenig Glück auch, was die Gelegenheiten betrifft, bestimmt. Wichtig sei, so Lippmann weiter, dass er selbst etwas dazu tue. Er habe sich schon immer für die Individualität im Menschen interessiert. ${ }^{73}$ Ein solches Empfinden verrät ,tief-bürgerliches' Selbstverständnis. Insbesondere lässt er eine innere Distanz zum bestehenden System erkennen, betont dennoch Disziplin: $\mathrm{Ob}$ man sich woblfüblt und Freude an einer Sache hat, ist ja in erster Linie eine Frage der konkreten gesellschaftichen Beziehungen. Wie Sie wissen, bin ich parteilos. Aber ich bin Hochschullebrer, ich bin Bürger dieses Staates, ich füble mich ibm nicht weniger verpflichtet als jeder Angehörige einer Partei. Deshalb auch nebme ich die Funktion eines Vorsitzenden ernst. ${ }^{74}$

Auch im Falle des „Carus-Clubs“ sind es wieder zentrale Figuren, die ,bildungsbürgerliche Lebenshaltung` sowohl vorleben als auch zu vermitteln versuchen, wobei Hans-Georg Lippmann als ein Protagonist erkannt werden kann. Seine Lebenshaltung prägte das Klubleben, auch nach seinem Ausscheiden als Vorsitzender, weiter, wurde adaptiert - zu erkennen an der Beibehaltung der Grußformel „Theuerste Freunde“ - sowie am kulturellen CEuvre der folgenden Jahre. Bei der Programmgestaltung hatten die Verantwortlichen relativ große Gestaltungsspielräume. Seitens des Kulturbundes wurde wenig ,hineindirigiert‘. Dem Klub wurde ein Instrukteur zugeordnet, der stets anwesend war, später noch ein Sekretär - beide nahmen auch an den Vorstandssitzungen teil, hätten aber wenig dirigistisch gewirkt, sondern zum Teil auch belfend, unterstützend und jedenfalls auf unsere Arbeit haben die wenig Einfluss genommen, erinnert sich ein Mitglied des Vorstandes.

Vermochte der „Carus-Club“ zwischen 1984 und 1989 als Institution des Dresdner Kultur-, Wissenschafts- und Geisteslebens eine integrative Wirkung zu entfalten, so verlor er diese im Zuge der politischen ,Wende' nahezu schlagartig. Vor allem ein nunmehr tatsächlich erforderliches, bis dahin mehr oder minder erfolgreich umgehbares, politisches Bekennen mag hier als ein wesentlicher Grund anzusehen sein. Es ließe sich von einer zuvor bestehenden ,Interessenskohäsion‘, vor allem über das integrierende Moment, bürgerlicher Hochkultur ‘ sprechen, die durch ,politische Dotierung' entkräftet wurde. Auf diesen Aspekt und andere Überlegungen verweist der nun folgende, letzte Abschnitt.

72 „Festlich - Nichts weiter“ (wie Anm. 70).

73 Vgl. ebd.

74 Ebd. 
Michael Kunz

\section{Resümee und Deutung}

Die Ergebnisse der Forschungsarbeit über die Institutionen der Dresdner Intelligenz lassen sich in insgesamt vier Kategorien verdichten, wenngleich sie nicht trennscharf zu behandeln sind. Es soll versucht werden, die Forschungsergebnisse anhand nachstehender Kategorisierung zu strukturieren: a) allgemeine Hochschätzung ,bürgerlicher 'Tugenden und Kultur; b) Formen der bürgerlichen Organisation; Selbstrekrutierungseffekte; c) Entpolitisierungstendenzen in den Institutionen; ,Interessenskohäsionen' und d) Einfluss und Wirkung zentraler Persönlichkeiten.

a) Die allgemeine Hochschätzung ,bürgerlicher 'Tugenden und Kultur

Verschiedentlich von Zeitzeugen angesprochen und im überlieferten Schriftgut erkennbar sind Haltungen, die mit den Merkmalen ,Bürgerlicher Kultur' stark korrelieren: als zentrale Punkte lassen sich der Wunsch nach Freiheit und ,Freizügigkeit', hoher persönlicher Bildungsanspruch, ein ausgeprägtes Interesse an der ,Hochkultur' und Traditionspflege erkennen. Insbesondere auch das Moment der ,Geselligkeit" war ein Grundanliegen, das schon von Ardenne in seiner Eröffnungsrede als Attraktion des „Dresdner Klubs“ einforderte. ${ }^{75}$

Prof. Hans-Georg Lippmann etwa, der Initiator des „Carus-Clubs“, verwies im Interview auf den Anspruch, nicht einfach Wissen [zu] vermitteln oder einen Vortrag mit etwas Musik [zu], umrabmen'. In der Aussprache baue man auf Mitdenken, im musikalischen Teil dagegen auf ein Ansprechen der Emotionen. ${ }^{76}$

Insbesondere im "Carus-Club" versuchte man nun gezielt auch an die bürgerlichen Traditionen des frühen 19. Jahrhunderts anzuknüpfen. Dabei wurde die Universalität des Namenspatrons Carl Gustav Carus als handlungsweisend erachtet, verbunden mit einem - hier von Hans-Georg Lippmann der Ärzteschaft zwar unterstellten, doch allgemein als vorhanden erachteten - Interesse an ,Hochkultur', denn es hätten Ärzte traditionell in der Regel besonders enge Beziehungen zu den Künsten (Musik, Bildende Kunst, Darstellende Kunst), die zu einem wesentlichen Teil nicht nur den Freizeitinhalt bestimmen, sondern auch in die Arbeitssphäre hineinreichen. ${ }^{77}$ Dies wurde sogar in der Satzung verankert. ${ }^{78}$

Auch der persönliche Anspruch an Bildung und Leistung wird wiederholt herausgehoben. Dabei bezog sich Prof. Lippmann sowohl auf fachliche als auch auf kulturelle Bildungsinhalte. Besonders prägnant kommt dies aber bei Manfred von Ardenne zum Ausdruck, der hier zudem eine methodische Lebensführung einforderte, als er postulierte, nicht die eigene Herkunft, sondern die Größe der Leistung sei maßgeblich. ${ }^{79}$

\footnotetext{
75 Von Ardenne, Gedanken (wie Anm. 32), S. 1.

$76 \mathrm{Vgl}$. „Festlich - Nichts weiter“ (wie Anm. 70).

77 Bezirks-Aktionsprogramm (wie Anm. 65), S. 2

$78 \mathrm{Vgl}$. Satzungen (wie Anm. 68).

79 Von ARdenne, Gedanken (wie Anm. 32), S. 3.
} 
,Freiheit‘ wurde ebenfalls thematisiert, mitunter auch im Zusammenhang mit beruflicher Qualifizierung, was sich etwa im Wunsch nach Zugang zu westlicher Fachliteratur äußerte. Für von Ardenne war dies beispielsweise wesentliche Attraktion des „Dresdner Klubs“. ${ }^{80}$ Als Beschränkung jedenfalls empfand man die politische Bevormundung, zielte vielmehr auf das Gespräch zu zweit oder zu dritt, möglichst in völlig zwanglosem Rabmen als die wabre Quelle wichtigster Anregung. ${ }^{81}$,Freizügigkeit' nahm man nun auch in Form von Privilegien wahr, so etwa der Möglichkeit, im klubeigenen Restaurant des Lingner-Schlosses ungezwungen und tageszeitunabhängig zu speisen, ohne übliche Beschränkungen (Platzierung, Reservierung etc.).

b) ,Bürgerliche‘ Formen der Organisation; Selbstrekrutierungseffekte

,Autonomie، und ,Autokephalie‘ sind Indikatoren dafür, inwieweit ein Verband seine eigene Ordnung satzen kann und inwieweit Chancen bestehen, den ,Leiter oder den ,Verwaltungsstab“ nach eigener Ordnung zu bestellen. ${ }^{82}$ Folgt man der Weberschen Definition eines Verbandes, ergibt sich, übertragen auf die Dresdner Institutionen, folgendes Bild: Die Wahl der Vertretungen der verschiedenen Klubs beruhte auf ,demokratischen Prinzipien', zumindest waren Wahlen und Abwahl möglich, auch wenn sich ,Auswahl‘ auf Einheitslisten beschränkte. Dass ,Instrukteure ${ }^{6}$ und ,Sekretäre' des Kulturbundes der Klubleitung beigestellt waren („Carus-Club“) oder wie im Falle des „Dresdner Klubs“ auch hohe SED-Funktionäre mit Mitgliederstatus, hatte wohl nur geringfügige Auswirkung auf die Gestaltung des Klublebens. Dem Kuratorium des „Dresdner Klubs“ wurden erhebliche Mitspracherechte eingeräumt. So war beispielsweise ein von der SEDParteigruppe gefordertes neues Statut für den „Dresdner Klub“ nicht ohne Zustimmung dieses Gremiums durchsetzbar. ${ }^{83}$

Betrachtet man die Satzung des „Carus-Clubs“, so fällt insbesondere die deutlich formulierte Zielsetzung einer Bereicherung des geistigen Lebens und [...] Entfaltung kultureller Interessen der medizinischen Intelligenz sowie der direkte Bezug auf das Wirken des Carl Gustav Carus auf. ${ }^{84}$ Politische Zielsetzungen hingegen beschränkten sich hier auf floskelhafte Füllsätze. Die große Übereinstimmung mit den von Hans-Georg Lippmann im Vorfeld formulierten Zielen eines solchen Klubs lassen vermuten, dass diese Satzung in relativer Eigenregie der Vereinigung entstanden war.

Es ist also festzuhalten: Die Vertretungsorgane wurden durch Wahl bestimmt. Einzig die Direktoren der Intelligenzklubs sowie die Sekretäre und Instrukteure

$80 \mathrm{Vgl}$. Brief von Ardenne an Oberbürgermeister Walter Weidauer (wie Anm. 33).

81 VON ARdENNE, Gedanken (wie Anm. 32), S. 3.

82 Vgl. Max Weber, Soziologische Grundbegriffe. Studienausgabe aus: Max Weber, Wirtschaft und Gesellschaft, Tübingen [1921], Tübingen 1984, S. 87.

83 Vgl. Bezirksleitung der SED, Abt. Kultur „Betr. Zuarbeit“ (Dossier zur Entwicklung speziell des „Dresdner Klubs“, Anm. M. K.)“, S. 4 (StA Dresden 13.50/Nr. 16).

84 Vgl. Satzungen (wie Anm. 68), S. 2. 
des Kulturbundes waren nicht frei bestellt, hatten allerdings auch keine Vertretungsfunktion nach außen hin und sind in diesem Sinne nicht Leiter oder, Verbandstab' zu nennen. Ihre Aufgabe war eine unterstützende oder ,geschäftsführende`, wenngleich mit bemessener Entscheidungsmacht verbunden.

Die Rekrutierung der Mitglieder gestaltete sich in den beiden Institutionen unterschiedlich. Die Anforderung an das persönliche Bildungsstreben, wie es beispielweise von Ardenne forderte, erwies sich als Distinktionsmerkmal, war gleichzeitig aber auch kennzeichnend für bildungsbürgerliche ,Rekrutierungsoffenheit'. Gerade im Falle des „Dresdner Klubs“ erhob man dieses zum Kriterium für eine ordentliche Mitgliedschaft. Hierfür wurden Rekrutierungs- und Exklusionsmechanismen eingeführt, die über einen längeren Zeitraum ein relativ geschlossenes ,Refugium' ermöglichten. Erleichterten Zugang erhielten demgegenüber Familienangehörige oder hinterbliebene Ehegatten. Ihnen wurde eine Mitgliedschaft im „Dresdner Klub“ ohne sonstige Forderungen offeriert. Hier ließe sich folgende Vermutung anstellen: Es kommt das bürgerliche Familienideal, das die Familie auch als ,kulturelle Einheit' betrachtet, zum Tragen; der „Dresdner Klub“ wird damit seiner Wirkung entsprechend vergleichbar einer ,Standesorganisation', die oftmals auch der Hinterbliebenen-Absicherung dient, im vorliegenden Fall einer ,kulturellen'.

Gerade die Einführung einer ,Empfehlungs- und Bürgenpraxis‘, mit anschließender ,Berufung', wie im „Dresdner Klub“, beschränkt sich nun in ihrer Wirkung wohl vor allem auf bestimmte Milieus; die geforderten Ansprüche an den Kreis der Mitglieder werden auf diese Weise tatsächlich durchgesetzt: Einerseits ist die Empfehlung von und das Bürgen für andere Personen in erster Linie tatsächlich eine ,Ehrensache', die mit der eigenen Reputation in Verbindung stehen kann, andererseits bleibt der Kreis von potenziellen Anwärtern hierdurch im Grunde eng umrissen.

Dies schloss aus oder wirkte zumindest erschwerend: $a$ ) auf bestimmte Alterskohorten, deren Angehörige aufgrund mangelnder beruflicher Entwicklungsmöglichkeiten noch keine adäquaten ,Leistungen' vorweisen konnten; $b$ ) weiterhin auf Personen, die in Hierarchie und Berufsstand unterhalb des im Klub organisierten ,durchschnittlichen Leistungsträgers' anzusiedeln waren; c) auf all jene, welche nicht in ähnlicher, ,milieutypischer' Weise über Bildungswissen verfügen konnten, insbesondere auf Frauen, die wohl aufgrund ihrer Ausbildungssituation im Jahre 1957 und noch danach im größeren Maße ausgeschlossen waren. Hieraus ergeben sich also nun zentrale Distinktionsmomente: Neben dem Anspruch, ,schöpferischer Leistung' sind also auch der höhere akademische Grad, ein hoher (bürgerlicher) Bildungsanspruch sowie ausreichende bis hohe soziale und kulturelle Kompetenzen von entscheidender Bedeutung.

Anders als im „Dresdner Klub" war im „Carus-Club“ zwar ebenfalls eine schriftliche Empfehlung vorgesehen, ${ }^{85}$ doch wie aus Zeitzeugenberichten hervor-

85 Vgl. ebd., S. 5. 
geht, kam diese nie zum Tragen, da der ,Andrang' nie so groß gewesen sei. Die Verbandstärke blieb hier wohl im gewünschten Rahmen und war daher nicht auf Selektion angewiesen.

Systematisch wurden durch solche Rekrutierungsmechanismen aber auch die Bestrebungen der offiziellen Parteipolitik ausgehebelt, die Klubs der Intelligenz für eine neue, sozialistische Intelligenz zu öffnen. Dass die Einrichtung der Intelligenzklubs nur eingeschränkt Zustimmung erfahren hat, offenbart demgegenüber ihren subkulturellen (im hier verhandelten Sinne ,bildungsbürgerlichen') Charakter. Auch ideologische Barrieren - und diese könnten im Jahre 1957 noch in einem größeren Ausmaß vorhanden gewesen sein als im ,relativen' Pragmatismus der Endzeit der DDR - mögen für Vorbehalte gegenüber dieser Organisationsform lokaler ,Eliten' verantwortlich gewesen sein. Subkulturell sind sie auch deshalb zu nennen, weil die Organisation in diesen Klubs nicht ,handlungsleitend" war und somit nicht verbindlich für die Dresdner Intelligenz, auch nicht nur für ihre Spitzen. Jedoch verbanden sich damit erhebliche milieuinterne ,Kompetenzchancen'sowie ein Prestigegewinn, insbesondere ein „Berufsprestige', im besonderen Maße wohl im heterogeneren „Dresdner Klub“.

Zusammenfassend lässt sich festhalten: Die Selbstorganisation in verschiedenen Einrichtungen war für die Organisation im subkulturellen bildungsbürgerlichen Milieu Dresdens von großer Bedeutung. Das hier empfundene ,Insel'-Dasein, das sich bündelnde Kulturinteresse und die Möglichkeiten der Bildung von Netzwerken bewirkten eine ,Gesinnungs- und Interessenskohäsion', wie nachfolgend beschrieben. Eine solche gewinnt vor allem Bedeutung in einer Tendenz der ,Entpolitisierung'.

c) Entpolitisierungstendenzen; ,Gesinnungs- und Interessenskohäsionen“

Die Ausrichtung des „Dresdner Klubs“ erlaubt es tatsächlich, von einem ,geschlossenen Refugium` zu sprechen. Auch hier war die eben erwähnte, Entpolitisierung' des Klublebens zu beobachten, die sowohl durch Zeitzeugen als auch durch entsprechende Einschätzungen offizieller Parteikader dargestellt wird. In der SED-Zuarbeit wurde dies kritisch aufgegriffen und präzisiert: Mebrere Versuche, dem geistig-kulturellen Leben einen neuen Inhalt zu geben, mußten [...] immer wieder scheitern. Das Hauptinteresse der Mitglieder lag in der Nutzung der gastronomischen Einrichtung und im Rabmen des Klublebens im Besuch der geselligen Veranstaltungen, wie Modenschauen, Ausflügen, Weibnachts- und Silvesterfeiern, Faschingsveranstaltungen, Filmen aus dem kapitalistischen Ausland, Besuch der Herkuleskeule u. a. m. Deshalb wurde der Klub seinem eigentlichen Anliegen und seiner Aufgabe als Zentrum des wissenschaftlichen und politischen Meinungsstreites im Sinne der Beschlüsse von Partei und Regierung nicht mehr gerecht. ${ }^{86}$ An anderer Stelle wurde ausdrücklich auf die Aufnahmebedingungen als

86 Bezirksleitung der SED, Abt. Kultur „Betr. Zuarbeit“ (wie Anm. 83), S. 2. 
Hemmnis für eine politische Neuausrichtung hingedeutet: Das noch immer feblende neue Statut mit den neuen Aufnabmebedingungen und Beitragsregelungen hemmt die Gewinnung neuer Mitglieder, weil ohne diese eine inhaltliche Veränderung auf politisch-ideologischem Gebiet nicht möglich ist. ${ }^{87}$ Ohne neue Mitglieder - und das bedeutete, dass man hier offensichtlich auf linientreue Genossen setzte - war also politische Einflussnahme nur mäßig durchsetzbar. Die Folgen dessen wurden im Anschluss genannt: Die neue sozialistische Intelligenz hätte keine Beziehungen zu diesem Klub und seinen Mitgliedern finden können. Dies hätte zu einer Stagnation der Mitgliederzahlen und sogar zu einem leichten Rückgang geführt. Außerdem sei der Klub mit einem Durchschnittsalter von 57 Jahren überaltert. Auch könne das Neue und Fortschrittliche entsprechend den gesellschaftlichen Erfordernissen, die sich aus der kontinuierlichen Entwicklung unserer sozialistischen Gesellschaft ergäben, nicht grundsätzlich Fuß fassen. ${ }^{88}$ Offensichtlich vermied man es zudem auch nach Möglichkeit, Konflikte des Einzelnen mit dem politischen System in das Klubleben hineinzutragen.

Auch im „Carus-Club“ war eine solche Tendenz der „Entpolitisierung“ zu beobachten. Vordergründig rekrutierte sich hier das Gros der Mitglieder aus dem Milieu der Ärzteschaft, die in der DDR mit ihrer Tätigkeit ohnehin in relativer ,Politikferne' angesiedelt, allerdings unentbehrlich war. Der Kern der Arbeit dieses Klubs konzentrierte sich auf die Aktivierung und Wabrung geistig-kultureller Freizeit-Interessen der medizinischen und der sich ibr verwandt füblenden Intelligenz. ${ }^{89}$ Man vermied durch die Themenauswahl eine intensivere Auseinandersetzung mit der sozialistischen Gegenwartsgesellschaft, die allenfalls in Fachvorträgen thematisiert wurde.

Im vorliegenden Fall war es vor allem ein bestehendes, gemeinsames Interesse an ,bildungsbürgerlicher Kultur', das über die Institutionen der Intelligenz gemeinschaftlich bedient wurde. Die sich hierbei entwickelnden ,Kohäsionskräfte` wirkten gleichsam, solange die entsprechend notwendige ,Homogenität' hier eine ,kulturelle - vorhanden war. Änderten sich die Rahmenbedingungen und traten ,Interessens-Dotierungen', 90 etwa in Gestalt eines neues ,politischen Interesses' hinzu, so schwand diese soziale ,Kohäsionskraft' als eine vereinende. Damit sei nicht gemeint, dass sich etwa das (hier: kulturelle) Interesse eines Klientels grundsätzlich änderte, lediglich als ,gemeinsame Basis‘ wurde es in Frage gestellt. Beim „Dresdner Klub“ etwa lag ein solches gemeinsames Interesse vor, gerade weil sich hier ein besonderes Milieu zusammenfand, in dem nun ,Kompetenzchancen'von besonderer Bedeutung waren.

87 Ebd., S. 3.

88 Vgl. ebd., S. 2 f.

89 Einladungs- und Informationsschreiben „Theuerste Freunde“ (wie Anm. 58), S. 2.

90 Dieser Terminus ist eigentlich der Physik entlehnt und bezeichnet dort das Einbringen fremder Teilchen in einen reinen Stoff. Im hier gemeinten Sinne soll ,Dotierung' als Auftreten von ,neuen` oder ,fremden' Interessen innerhalb einer Gemeinschaft verstanden werden. 
Insbesondere aber beim „Carus-Club“ lässt sich dieser Effekt recht gut nachvollziehen. Wie ein Zeitzeuge im Interview mit dem Verfasser bemerkte, seien bei Ärzten oftmals politische Dinge binten angestellt worden. Konzentriert habe man sich auf die Arbeit. So wären in der DDR von Ärzten kaum einschneidende politische Zugeständnisse gemacht worden. Hinzu kam ein unter Ärzten hoch angelegtes berufliches Ethos, das einherging mit Tendenzen einer ,Entpolitisierung' sowohl des beruflichen, als auch des privaten Umfeldes. ${ }^{91} \mathrm{Im}$ Zuge politischer Öffnung in der Wendezeit verliert der „Carus-Club“ schließlich seinen Refugiumscharakter, gerade da es nunmehr unumgänglich erschien, sich auch politisch zu positionieren. Die ,politische Dotierung ' traf die Ärzteschaft im Besonderen und entsprechende ,Interessenskohäsionen', die hier im Vorfeld sehr stark ausgeprägt waren, verloren schlagartig ihre Bindungskraft.

\section{d) Einfluss und Wirkung zentraler Persönlichkeiten}

Die Dresdner Konstellation ist eine besondere: Es wurden wiederholt Personen angesprochen, die sich als zentral für die Konstituierung spezifischer Interessenskreise herausstellten. An dieser Stelle seien exemplarisch zwei der Protagonisten angeführt, deren Wirkung unmittelbar auf die besprochenen Institutionen der Dresdner Intelligenz abstrahlte: Manfred von Ardenne, sowie Karl Laux. Auf die Wirkung Lippmanns auf den „Carus-Club“ wurde bereits eingegangen.

Manfred Baron von Ardenne ${ }^{92}$ darf wohl die schillerndste und ambivalenteste Persönlichkeit der Dresdner Intelligenz genannt werden. Nicht nur verfügte er über das einzige privat geführte Forschungsinstitut der DDR mit nahezu 500 Angestellten, er unterhielt zudem bis in die frühen 1970er-Jahre auch exzellente Verbindungen in die höchsten Kreise der Politik (unter anderem zu Walter Ulbricht). Diese eigenwillige Position stellte ein Paradox im ansonsten streng hierarchisch gegliederten Machtgefüge der SED dar. Die Patronage von Ardennes endete mit der Absetzung Ulbrichts 1971 und dem neuen Kurs unter seinem Nachfolger Honecker, der wenig Verständnis für diese Art Sonderbehandlung aufbrachte. Besondere Bedeutung kommt von Ardenne bei der Gründung und Ausrichtung des „Dresdner Klubs“ zu. Er darf hier als federführend angesehen werden. Sein zweifelsohne bildungsbürgerlicher, ins großbürgerliche tendierender Habitus wirkte vorbildhaft, einerseits auf die alte Dresdner Intelligenz, andererseits auf jene, die in den späten 1940er- und frühen 1950er-Jahren leitende Positionen neu besetzten oder in diesen verblieben waren.

91 Uwe Tellkamp liefert mit seinem 2008 erschienenen Roman „Der Turm“ einen Einblick in das Dresdner Ärztemilieu der 1980er-Jahre. Recht eindringlich sind hier Befindlichkeiten und Alltag, aber auch die unverkennbare Politikferne eines zwar fiktiven, doch mit autobiografischen Einflechtungen versehenen, in relativer Abgeschlossenheit verharrenden Milieus beschrieben; Uwe Tellkamp, Der Turm, Frankfurt am Main 2008.

$92 \mathrm{Zu}$ seiner Person vgl. auch Gerhard Barkleit, Manfred Baron von Ardenne, in: Sächsische Biografie, hrsg. vom Institut für Sächsische Geschichte und Volkskunde, www. isgv.de/saebi (Zugriff 21. Juni 2012). 
Prof. Karl Laux ${ }^{93}$ war Rektor der Musikhochschule Dresden, Musiktheoretiker und -kritiker, bereits vor dem Zweiten Weltkrieg als Musikwissenschaftler tätig, von Beginn an Mitglied im „Dresdner Klub“, ebenfalls im Klub der Intelligenz „Victor Klemperer“ und schließlich im vereinigten „Dresdner Klub der Intelligenz", dem er neben von Ardenne als Ehrenvorsitzender vorstand. Zudem bekleidete er 1972 das Amt des Ersten Vorsitzenden des Deutschen Kulturbundes, Bezirksleitung Dresden. ${ }^{94}$ In dieser letztgenannten Position hatte er umfangreiche Einsicht und Mitspracherechte bei der Ausarbeitung und Umsetzung der Kulturbund-Programme für den Bezirk Dresden - mithin auch Möglichkeiten, auf die verschiedenen Klubs der Intelligenz einzuwirken, die ohnehin beim Kulturbund organisiert waren. Als eine zentrale Figur in der Dresdner Kulturszene konnte er weiterhin die Zusammenarbeit der Institutionen des Kulturbundes mit den Einrichtungen der Stadt arrangieren.

Die Wirkung von Einzelpersönlichkeiten kann in insgesamt drei Kategorien zusammengeführt werden. So ist eine solche festzustellen a) auf die Art der persönlichen Lebenshaltung durch spezifische Interessensvermittlung; $b$ ) auf die Schaffung von Freiräumen; schließlich c) auf die Bildung sozio-kultureller Netzwerke.

Dass die Art der persönlichen Lebenshaltung eines Umfeldes direkt oder indirekt tatsächlich durch Einzelpersönlichkeiten wesentlich beeinflusst werden konnte, lässt sich sowohl an von Ardenne als auch Lippmann erkennen. Mit der Ausgestaltung des Lingner-Schlosses als exklusives Klubhaus schuf ersterer die Örtlichkeiten, den Rahmen sozusagen für die spezifischen Interessen einer ausgesuchten Klientel. Die ,Attraktionen' des Klubs wurden so erst ermöglicht, vielleicht erst in dieser Form angeregt und sodann von den Mitgliedern des „Dresdner Klubs“ angenommen und übernommen, waren fortan persönliches Bedürfnis des Einzelnen. Ebenso konnte die programmatische Ausrichtung des Klubs, insbesondere wenn sie auf ein erkennbares Engagement Einzelner zurückging, sowohl Interessen bündeln als auch neue schaffen. Eine spezifische Ärztevereinigung wie der „Carus-Club“ vermag dies vielleicht noch gezielter, beispielsweise bei der Vermittlung bürgerlicher Ideale des 19. Jahrhunderts oder eines klassischen ,Bildungskanons', die selbst Aufnahme in die Satzung finden sollten. Hier wäre insbesondere auf Vorbildwirkungen zu verweisen. Ebenso waren Einzelpersönlichkeiten in die Lage versetzt, ,Freiräume' zu schaffen - politische wie auch kulturelle - soweit ihnen gewisse Gestaltungsmacht zustand. Ihr Wirken bestimmte, ,was möglich war', auch in Bezug darauf, ,wie weit man gehen konnte'. Im Rahmen dieser Institutionen unternahmen nun wiederum Einzelpersönlichkeiten zum Teil erfolgreich den Versuch, thematisch Neuland zu betreten und dieses für die übrigen Mitglieder zu erschließen. Solche Freiräume freilich waren fragil, was ein stetes Austarieren der aktuellen Interessen erforderlich machte, mitunter auch zu Arrangements zwang.

93 Vgl. auch Dieter Härtwig, Karl Laux, in: Sächsische Biografie (wie Anm. 92) (Zugriff 21. Juni 2012).

94 Dies geht aus einem Referat von Karl Laux hervor, das er auf der Bezirksdelegiertenkonferenz des Deutschen Kulturbundes am 3. Juni 1972 im Zuge der Vereinigungsdebatte beider Dresdner Intelligenzklubs hielt (vgl. StA Dresden 13.50/16; vgl. auch HStA Dresden 12723 Personennachlass Karl Laux). 